PANORAMA

ISSN: 1909-7433

ISSN: 2145-308X

ednorman@poligran.edu.co

Politécnico Grancolombiano

Colombia

\title{
LA PRESENCIA DE PHUBBING EN ESTUDIANTES DEL NIVEL SUPERIOR
}

Hernández Gracia, Tirso Javier; Duana Ávila, Danaé; Hernández Gracia, José Francisco; Torres Flórez, Dagoberto

LA PRESENCIA DE PHUBBING EN ESTUDIANTES DEL NIVEL SUPERIOR

PANORAMA, vol. 15, núm. 28, 2021

Politécnico Grancolombiano, Colombia

Disponible en: https://www.redalyc.org/articulo.oa?id=343965146005

Institución Universitaria Politécnico Grancolombiano

Institución Universitaria Politécnico Grancolombiano

\section{(c) $(1) \Theta$}

Esta obra está bajo una Licencia Creative Commons Atribución-NoComercial-SinDerivar 4.0 Internacional. 
Artículos de investigación científica y tecnológica

\title{
LA PRESENCIA DE PHUBBING EN ESTUDIANTES DEL NIVEL SUPERIOR
}

THE PRESENCE OF PHUBBING IN HIGHER LEVEL STUDENTS

\author{
A PRESENÇA DE PHUBBING EM ESTUDANTES DE \\ NÍVEL SUPERIOR
}

PANORAMA, vol. 15, núm. 28, 2021

Politécnico Grancolombiano, Colombia

Recepción: 08 Septiembre 2020

Aprobación: 21 Octubre 2020

Redalyc: https://www.redalyc.org/ articulo.oa?id $=343965146005$
Resumen: En el marco de la llamada cuarta revolución industrial, el internet y las TIC (Tecnologías de Información y Comunicación), se vuelven imprescindibles. Es decir, cada vez más la automatización de procesos y manejo de información por medio de dispositivos electrónicos se hace presente con mayor frecuencia, lo que implica contar con trabajadores mejor preparados para poder ser competitivos en sus empresas. En el caso de las instituciones educativas, los maestros y alumnos deben estar a tono con la evolución tecnológica y desarrollar competencias y habilidades que garanticen el proceso de enseñanza - aprendizaje. El objetivo de la presente investigación fue describir los distintos usos del celular por estudiantes universitarios, en un día de clases de una institución educativa tecnológica del centro de México. Los principales resultados obtenidos muestran que entre un $50 \%$ y $75 \%$ de los alumnos encuestados utilizan el teléfono celular en clase sin consentimiento del maestro, es decir, para atender asuntos personales.

Palabras clave: Phubbing, revolución industrial, universitarios, educación, tecnología educacional, tecnología de la información.

Abstract: In the framework of the so-called fourth industrial revolution, the internet and ICT (Information and Communication Technologies), become essential. In other words, the automation of processes and information management through electronic devices is increasingly present, which implies having better prepared workers to be able to be competitive in their companies. In the case of educational institutions, teachers and students must be in tune with technological evolution and develop competencies and skills that guarantee the teaching - learning process. The objective of this research was to describe the different uses of the cell phone by university students, in a class day at a technological educational institution in central Mexico. The main results obtained show that between $50 \%$ and $75 \%$ of the surveyed students use the cell phone in class without the teacher's consent, that is, to attend to personal matters.

Keywords: phubbing, industrial revolution, university students, education, educational technology, information technology. 
Resumo: No quadro da chamada quarta revolução industrial, a Internet e as TIC (Tecnologias de Informação e Comunicação) tornam-se essenciais. Ou seja, a automatização de processos e gestão da informação através de dispositivos eletrónicos está cada vez mais presente, o que implica ter trabalhadores mais preparados para serem competitivos nas suas empresas. No caso de instituições de ensino, professores e alunos devem estar em sintonia com a evolução tecnológica e desenvolver competências e habilidades que garantam o processo de ensino - aprendizagem. O objetivo desta pesquisa foi descrever os diferentes usos do telefone celular por estudantes universitários, em um dia de aula em uma instituição de ensino tecnológico na região central do México. Os principais resultados obtidos mostram que entre $50 \%$ e $75 \%$ dos alunos pesquisados usam o celular em sala de aula sem o consentimento do professor, ou seja, para atender a assuntos pessoais.

Palavras-chave: phubbing, revolução industrial, estudantes universitários, Educação, tecnologia educacional, tecnologia da informação.

\section{INTRODUCCIÓN}

Con la evolución acelerada de la tecnología en todos los ámbitos, incluyendo por supuesto el educativo, es necesario formar un frente común entre maestros, alumnos y directivos, cuyo propósito sea el de plantear soluciones que permitan preparar a los próximos profesionales con capacidades, habilidades, conocimientos y competencias para enfrentar el mundo del trabajo en la bien llamada "cuarta revolución industrial”, donde el uso intenso del internet cada vez gana más terreno en las nuevas generaciones, al igual que la automatización de los procesos en las empresas, instituciones y todo tipo de organizaciones públicas. Esto involucra también que la mano de obra sea más especializada, teniendo implicaciones económicas y sociales, ya que no se puede eludir el fenómeno que desde la primera revolución industrial ha traído consigo "el desplazamiento o despido de trabajadores", por la puesta en operación de millones de computadoras, robots y máquinas capaces de realizar funciones multitarea con mayor rapidez y precisión que el mismo ser humano.

El fin de la presente investigación no es analizar las repercusiones económicas y sociales derivadas de la cuarta revolución industrial en términos de empleo o desempleo, más bien es direccionar el efecto que está causando en los estudiantes el abuso y mal uso de las tecnologías, trayendo consigo una falta de sensibilidad y disminución de valores durante los procesos de comunicación que se llevan a cabo en el aula, principalmente con el maestro. Aunque todo ello también lo ha trasladado el estudiante al terreno familiar y empresarial, cuando participa en reuniones familiares y realiza sus prácticas y servicio social, respectivamente.

Este fenómeno es conocido como phubbing, que se puede traducir como 'ningufoneo', es decir, ignorar a una persona por estar concentrada su atención en el uso de una tecnología portátil, como lo es el celular o tableta. El alumno de hoy abusa de la tecnología en momentos impropios, es decir, cuando no debe utilizarlos y concentrarse en la comunicación que está recibiendo por parte del maestro en el aula. 
El objetivo del estudio fue describir los distintos usos del celular por estudiantes universitarios, en un día de clases de una institución educativa tecnológica del centro de México.

Los principales resultados obtenidos muestran que entre un $50 \%$ y $75 \%$ de los alumnos encuestados utilizan el teléfono celular en clase sin consentimiento del maestro, es decir, para atender asuntos personales.

\section{Comunicación y tecnoferencia}

La comunicación es fundamental dentro del proceso enseñanza aprendizaje, ya que debe garantizar su fin u objetivo, es decir, no basta con transmitir los conocimientos de un maestro a un alumno, sino provocar el acto de comprensión donde participan ambos sujetos. Toda institución educativa, privada o pública, debe colaborar para que dicho acto se cumpla, desde el proceso pedagógico hasta el currículo de las asignaturas, potenciando conocimientos y habilidades, a partir de diversos escenarios y situaciones que enfrenta el alumno, tomando en cuenta los materiales y elementos tecnológicos necesarios (Vargas, Fernández \& Bauza, 2016).

Se sabe que actualmente los medios de comunicación han evolucionado ampliamente, lo cual se convierte en un desafío para la educación, e impulsa a repensar el fenómeno y establecer estrategias por parte de los maestros, principalmente para que los alumnos utilicen de manera adecuada y eficiente las Tecnologías de Información y Comunicación (TIC), pues el nivel de dependencia que ha generado en el ser humano en general es muy alto (Hertlein \& Blumer, 2014), lo que ha traído consigo también momentos de intrusión o interferencia (tecnoferencia) entre los sujetos que participan en el acto de la comunicación (González et al., 2017).

El ignorar a otra persona por estar utilizando un dispositivo digital, como puede ser un smartphone, computadora, tableta, entre otros dispositivos móviles que hoy en día existen en el mercado, se llama tecnoferencia. Todo ello pareciera una paradoja, ya que "uno de los beneficios del uso de las TIC es que nos acercan a quienes están lejos, pero uno de sus peligros es que nos alejan de quienes tenemos más cerca" (Capilla, 2017, p. 117).

Es claro que, en el nuevo contexto social emergente, es indispensable la utilización de diversos dispositivos móviles digitales, ya que son parte de la educación 4.0, lo cual implica para el alumno el desarrollo de nuevas potencialidades y habilidades para el aprendizaje de conocimientos en diversos ámbitos académicos. Todo ello es plausible y necesario, puesto que los jóvenes de hoy provienen de generaciones que nacieron con el internet y la red, pero también existen riesgos detrás de todo este entramado tecnológico, que es la pérdida de valores asociados a la falta de atención, un acto descortés hacia los presentes (Delena, 2014).

En el aula es común que los alumnos, no solo del nivel licenciatura, sino también los de posgrado, se distraigan de manera continua durante la clase, porque aunque el maestro se los prohíba ven la manera de sacar el dispositivo a escondidas y estar revisando sus mensajes, o bien en 
clases donde se les permite usar la tableta o computadora, se conectan a la red para tener abiertas al mismo tiempo diversas pestañas en el navegador y así interactuar de manera multitasking. Adicionalmente, existen diversos escenarios donde la constante es la misma, ya que cuando un conglomerado de estudiantes es invitado a asistir a una conferencia o cualquier otro evento académico, con el pretexto de tomar fotografías se distraen del objetivo principal para el que fueron convocados.

\section{Concepto de phubbingy problemas asociados}

No se pretende minimizar la importancia de la sociedad virtual, ya que se reconoce que las instituciones promueven su oferta educativa en diversas modalidades, además de la constante renovación de sus aulas y escenarios de aprendizaje donde destacan el uso de tecnologías de vanguardia, así como la educación continua que reciben sus docentes para estar mejor preparados en la utilización de materiales didácticos modernos. Hoy en día un alumno que no interactúa con sus compañeros y maestros mediante diversos dispositivos tecnológicos está condenado a ser un ente aislado o marginado, con pocas posibilidades de poderse adaptar al mundo educativo actual. No obstante, el abuso y mal uso de los diversos dispositivos electrónicos provoca una adicción que muchas veces tiende a caer en la exageración, desencadenando una serie de actitudes negativas que impiden la comunicación entre diversos sujetos. Como ya se ha comentado antes, es común que este tipo de situaciones se presente de manera recurrente en un salón de clases, mientras el maestro se encuentra impartiendo su cátedra o un equipo de alumnos expone un tema relacionado con la asignatura que se cursa. Aunque existen diversos dispositivos que se convierten en distractores, el que más destaca es el teléfono móvil.

En el mundo anglosajón se le conoce a este fenómeno como phubbing, de ahí que sea un término formado por dos palabras phone, que significa teléfono, y snubbing, que quiere decir despreciar. Gómez define este constructo como "el acto de menospreciar a quien nos acompaña al prestar más atención al móvil u otros aparatos electrónicos que a su persona" (2013, p. 25). Según diversas investigaciones, este término apareció por vez primera en Australia, de manera más específica en el año 2013, al referirse a la conducta de ignorar a otra persona durante una conversación por encontrarse operando un teléfono celular, lo cual provoca interferencia en la comunicación (Barrios et al., 2017).

La estructura del phubbing es multidimensional, toda vez que los dispositivos celulares aglutinan en su interior diversas funciones, es decir, a pesar de que el propósito fundamental es el realizar llamadas telefónicas entre una o más personas, también ofrecen servicios como internet, redes sociales (WhatsApp, Facebook, correos, Twitter, Instagram, etc.), videojuegos, páginas web con propósitos específicos como salud, moda, esparcimiento, entre otros. Es precisamente cuando se tiene acceso a ese tipo de información, en situaciones en las que no son las más propicias para hacerlo, se genera una falta de atención o distracción. 
El phubbing trae consigo una serie de consecuencias, por eso es importante resaltar los problemas que puede ocasionar la adicción al celular, ya que lamentablemente se tienen casos documentados donde este tipo de distracciones han repercutido severamente en accidentes laborales, de tránsito, problemas de pareja o familiares, efectos en la salud (estrés, depresión, ansiedad, comportamientos agresivos, nerviosismo, angustia, nomofobia, entre otros).

La nomofobia, de acuerdo con Félix et al. (2017, p. 24), es: "el miedo irracional a salir de casa sin un teléfono móvil...es un desorden psicológico muy común, más en los jóvenes". Este concepto es más amplio, ya que también refiere a la sensación de sentirse desconectado del mundo en general. Es precisamente este tipo de sensaciones que los alumnos llegan a sentir, no solo al saber que su teléfono celular puede quedar sin batería durante su jornada escolar, sino la tentación de querer ver a cada rato sus mensajes o aplicaciones adicionales mientras están en clase.

El uso del teléfono celular en clase es importante como parte del modelo educativo fundamentado y complementado en el uso de espacios virtuales, mediante diversas técnicas como e-learning, m-learning, blearning, entre otras, donde el aprendizaje abarca diversas dimensiones (presencial, online y mixto). Schwab (2016), al igual que diversos autores, plantean precisamente la necesidad de enfrentar hoy en día la cuarta revolución industrial, que se distingue por la incorporación de nuevas tecnologías que fusionan la parte física, digital y biológica, afectando todas las disciplinas y campos de estudio. El que un alumno busque en clase, con asistencia del maestro, información sobre algún concepto o campo académico es muy importante, ya que el teléfono celular se convierte en una valiosa herramienta de apoyo didáctico y solución de tareas, pero si en lugar de darle ese sentido los alumnos aprovechan cualquier momento para distraer su atención del objetivo académico, entonces esa realidad se convierte con el tiempo en exámenes reprobados, sanciones de todo tipo, temas difíciles de comprender y estudiar, apuntes incompletos, materias no acreditadas, entre otros aspectos.

Es claro que el phubbing en la era de educación 4.0 genera un impacto sobre la satisfacción de las relaciones alumno-maestro, su rendimiento escolar y la salud mental de los propios estudiantes. Es por ello, que en el presente estudio se decidió ir más allá de un análisis teórico y así poder llevar a cabo un primer acercamiento con una realidad concreta en alumnos del nivel universitario de una universidad tecnológica del centro de México.

El objetivo fue describir los distintos usos del celular por estudiantes universitarios de una institución educativa tecnológica del centro de México en un día de clases. La metodología utilizada fue descriptiva y la recolección de datos cuantitativa. La población estuvo compuesta por 120 alumnos del quinto y octavo semestre de la Licenciatura en Inteligencia de mercados y psicología, quienes aceptaron participar, obteniendo una muestra de 92 sujetos, misma que fue calculada mediante una fórmula para poblaciones finitas, utilizando una máxima variabilidad, un nivel de confianza del $95 \%$ y un error permisible del $5 \%$. 


\section{METODOLOGÍA}

El instrumento utilizado fue un cuestionario diseñado por los autores de la presente investigación, tomando como base los desarrollados en otros estudios similares como Cisneros y Robles (2017), Capilla y Cubo (2017) y Villafuerte y Vera (2019), entre otros autores. En una primera sección se aglutinaron algunas preguntas generales como edad, género, tipo de alumno, promedio escolar, turno, entre otras. En una segunda sección se plantearon ítems de diversas preguntas en una escala de Likert de 1 a 5 , donde $1=$ nunca, $2=$ casi nunca, $3=$ a veces, $4=$ casi siempre y $5=$ siempre, agrupadas en tres dimensiones o categorías: A. Uso del teléfono (10 preguntas), B. Problemas del uso del teléfono (10 preguntas), C. Sentimiento de culpa ( 5 preguntas). Se calculó la fiabilidad del instrumento, para lo cual se utilizó el coeficiente de Alpha de Cronbach, obteniendo un valor de 0.81 .

Alguna de las preguntas iniciales del tipo sociodemográficas se utilizaron únicamente para realizar correlatos con cada una de las tres dimensiones del instrumento para medir el phubbing.

Hipótesis del estudio

- H1: Al menos un 30\% de los alumnos participantes en el estudio utilizan el teléfono celular en clase sin consentimiento del maestro, es decir, para atender asuntos personales.

$\mathrm{H} 2$ : Existe una relación positiva entre las dimensiones del phubbing y el turno en que cursan sus materias los estudiantes universitarios de esta universidad tecnológica del centro de México.

H3: El tipo de alumno se encuentra fuertemente asociado con cada una de las dimensiones del phubbing.

H4: Las relaciones de noviazgo/pareja que tienen los estudiantes universitarios de esta universidad tecnológica del centro de México se encuentra asociada positivamente con cada una de las dimensiones del instrumento de phubbing.

\begin{tabular}{|r|l|}
\hline Características generales & Porcentajes y promedios \\
\hline Tamaño de la muestra = 92 casos \\
\hline Género & $82,1 \%$ son mujeres (76 casos) \\
\hline Edad & 20 a 22 años $(53,6 \%-49$ casos) \\
\hline Turno & $64,3 \%$ (59 casos) vespertino. \\
\hline Tipo de alumno & $85 \%$ (78 casos) son alumnos regulares \\
\hline Número de asignaturas que cursa & $53,6 \%$ (49 casos) cursa 6 materias. \\
\hline Promedio general & 8,2 \\
\hline Relaciones de familia & $90 \%$ (83 casos) tienen buena relación. \\
\hline Relaciones de noviazgo/pareja & $\begin{array}{l}42,9 \% \text { (39 casos) tienen buena relación, el 25\% (23 } \\
\text { casos) regular y el resto no tiene novio(a). }\end{array}$ \\
\hline Preocupaciones personales & $\begin{array}{l}82 \% \text { (76 casos) determinaron sentir algún tipo de } \\
\text { preocupación, siendo la familia la principal y de otro tipo. }\end{array}$ \\
\hline Residencia & $\begin{array}{l}53,6 \% \text { (49 casos) no viven en el mismo lugar donde } \\
\text { estudian, por lo que tienen que viajar. }\end{array}$ \\
\hline &
\end{tabular}

Tabla 1

Características generales de los estudiantes universitarios

Fuente: elaboración propia con base en los resultados de la investigación. 


\section{RESULTADOS}

Se puede apreciar en la tabla 1 los datos generales obtenidos de la encuesta aplicada, donde la mayoría son mujeres, cuyas edades fluctúan entre los 20 y 22 años y las tres cuartas partes estudian por la tarde. Llama la atención que, al ser la mayoría del sexo femenino, pudiera pensarse que son las que más tienden -por su naturaleza- a establecer pláticas telefónicas activas. También el turno podría favorecer el mayor uso del teléfono celular, ya que es más común que por la tarde haya mayor disponibilidad de las personas para establecer comunicación con sus semejantes. El número de alumnos regulares en principio es alto, aunque quizás en futuras investigaciones se podría considerar las asignaturas reprobadas o exámenes extraordinarios presentados. Finalmente, el porcentaje de preocupación es alto, lo cual podría estar asociado con la necesidad de comunicarse con frecuencia para disipar de alguna manera sus preocupaciones. Este es, en principio, un panorama general de algunas variables sociodemográficas que van a permitir tener un primer acercamiento y reflexión de los datos.

Enseguida, se presenta en la tabla 2 una serie de datos estadísticos descriptivos, basados en las medidas de tendencia central, para ir desmenuzando con ello algunos otros análisis importantes relacionados con las tres dimensiones del instrumento phubbing.

\begin{tabular}{|l|c|c|c|}
\hline \multirow{2}{*}{ Estadísticas descriptivas } & $\begin{array}{c}|c| \\
\text { Dso del } \\
\text { teléfono }\end{array}$ & $\begin{array}{c}\text { Problemas del uso del } \\
\text { teléfono }\end{array}$ & $\begin{array}{c}\text { Sentimiento de } \\
\text { culpa }\end{array}$ \\
\hline Media & 3.20 & 1.97 & 1.94 \\
\hline Mediana & 3.15 & 1.80 & 1.60 \\
\hline Moda & 2.88 & 1.40 & 1.40 \\
\hline Desviación estándar & 1.01 & 1.08 & 1.10 \\
\hline
\end{tabular}

Tabla 2

Medidas de tendencia central del phubbing en estudiantes universitarios

Fuente: elaboración propia con base en los resultados de la investigación.

Se puede observar que las puntuaciones medias más altas se obtuvieron en la dimensión correspondiente al uso del teléfono con 3.20, mientras que las otras dos dimensiones, problemas del uso del teléfono y sentimiento de culpa fueron de 1.97 y 1.94 , respectivamente. Esto significa que la frecuencia con que utilizan el celular los estudiantes universitarios se ubica entre la opción a veces y casi siempre, es decir, si consideramos que en la escala de Likert la opción 1 vale $0 \%$ y la 5 el 100\%, entonces los valores obtenidos oscilarían entre el 50 y $75 \%$. Con esto la hipótesis H.: Al menos un 30\% de los alumnos participantes en el estudio utilizan el teléfono celular en clase, sin consentimiento del maestro, es decir, para atender asuntos de manera personal, se acepta. 


\begin{tabular}{|l|c|r|c|c|}
\hline Dimensiones del phubbing / turno & Media & N & Prueba F & \multicolumn{1}{|c|}{ p } \\
\hline Uso del teléfono & & & 20.010 & 0.020 \\
- Vespertino & 3.3389 & 59 & & \\
- Mixto & 2.9400 & 33 & & \\
\hline Problemas del uso del teléfono & & & 0.171 & 0.822 \\
- Vespertino & 2.0000 & 59 & & \\
\hline Mixto & 1.9300 & 33 & & \\
\hline Sentimiento de culpa & & & 2.210 & 0.420 \\
- Vespertino & 2.0333 & 59 & & \\
- Mixto & 1.7600 & 33 & & \\
\hline
\end{tabular}

Tabla 3

Puntuaciones medias del phubbing con el turno

Fuente: elaboración propia con base en los resultados de la investigación.

En la tabla 3 se puede observar que las puntuaciones medias más altas corresponden a la dimensión uso del teléfono (media=3.3389), con un nivel de significancia de 0.020 , lo que puede interpretarse como una relación positiva con el turno vespertino que cursan los estudiantes universitarios. No obstante, con las dimensiones problemas del uso del teléfono y sentimiento de culpa, no existe una correlación positiva. Es por ello por lo que la hipótesis establecida H.: Existe una relación positiva entre las dimensiones del phubbing y el turno en que cursa sus materias los estudiantes universitarios de una universidad tecnológica del centro de México, se acepta parcialmente, es decir, solo con la primera dimensión del instrumento de phubbing utilizado.

En la tabla 4 se localizan los datos derivados de los correlatos obtenidos entre las tres dimensiones del phubbing y el tipo de alumno. Las puntuaciones medias más altas corresponden a la dimensión uso del teléfono (media=3.2074), con un nivel de significancia de 0.010; así mismo, la segunda dimensión relacionada con problemas del uso del teléfono arroja datos de media $=1.9889$ y $p=0.027$, mientras que la tercera dimensión que hace referencia a sentimiento de culpa reflejó no estar asociada. Todo esto significa que, de acuerdo con la hipótesis planteada: H.: El tipo de alumno se encuentra fuertemente asociado con cada una de las dimensiones del phubbing, se acepta parcialmente, toda vez que la variable tipo de alumno se encuentra asociada fuertemente con dos de las tres dimensiones del phubbing.

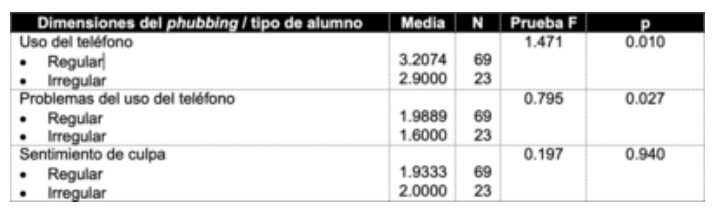

Tabla 4

Puntuaciones medias del phubbing con el tipo de alumno

Fuente: elaboración propia con base en los resultados de la investigación.

Finalmente, en la tabla 5 se correlacionaron las dimensiones del phubbing con la variable denominada relaciones de noviazgo/pareja que pudieran o no tener los estudiantes universitarios que participaron en el estudio. Los resultados muestran que existe una asociación positiva entre el uso del teléfono (media $=3.2750$ y $\mathrm{p}=0.030$ ), problemas del uso del teléfono (media $=2.1833$ y $\mathrm{p}=0.042$ ) y sentimiento de culpa 
(media $=2.0833$ y $\mathrm{p}=0.001$ ). La hipótesis $H .:$ Las relaciones de noviazgo/ pareja que tienen los estudiantes universitarios de una universidad tecnológica del centro de México se encuentra asociada positivamente con cada una de las dimensiones del instrumento de phubbing, se acepta.

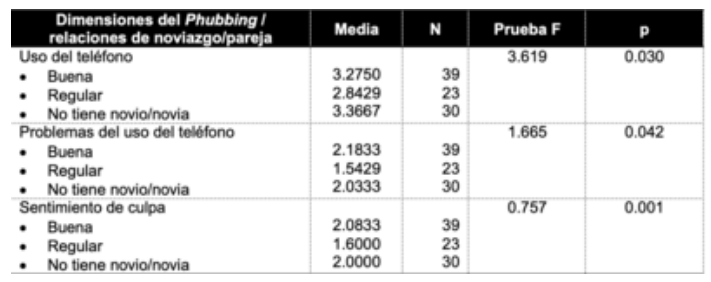

Tabla 5

Puntuaciones medias del phubbing con las relaciones de noviazgo/pareja

Fuente: elaboración propia con base en los resultados de la investigación.

\section{DISCUSIÓN}

La utilización del teléfono celular en clase es indispensable hoy en día para un estudiante universitario, sobre todo como herramienta de aprendizaje para la búsqueda de información, operación de plataformas y software especializado, redes sociales, entre otras, pero todas ellas enfocadas a potenciar sus conocimientos en el aula y bajo la supervisión del profesor. Capilla (2017) expone que existe una relación entre el uso de la telefonía móvil y el bienestar psicológico; así, se puede interpretar que los estudiantes que continuamente se encuentran utilizando su teléfono celular a todas horas, incluyendo el salón de clase, pueden presentar sensaciones derivadas de aspectos asociados con su salud, relaciones con personas, estatus académico, distracción y ocio, entre otros, es decir, el efecto puede producir un bienestar psicológico positivo o negativo. Los resultados obtenidos reflejan que existe de alguna manera una asociación entre el uso del dispositivo telefónico móvil con algunos aspectos situacionales presentes o pasados del estudiante universitario, como el ser un alumno regular o no, el haber tenido algunos problemas en la escuela o la familia, así como dolor de cuello, manos, visión cansada, entre otros aspectos.

En futuros estudios se deben analizar con profundidad algunas variables que requieren su abordaje de manera longitudinal como el turno, promedio general, hábitos de estudio, género, entre otras, de los estudiantes universitarios, ya que quizás se conviertan en facilitadoras del uso excesivo e inadecuado del celular en el salón de clase, así como en otros escenarios sociales.

\section{CONCLUSIONES}

Es importante reconocer que el phubbing es un fenómeno que interfiere la comunicación en el aula y afecta de algún modo al estudiante universitario de manera personal en su bienestar psicológico, y trae consecuencias a la 
salud y las relaciones sociales, por lo que se deben buscar estrategias para su atención y utilización de manera responsable.

La educación 4.0 trae consigo nuevos modelos educativos, basados principalmente en la utilización de tecnología moderna; no obstante, debemos reconocer la realidad social que actualmente se vive con los alumnos de todos los niveles educativos, pero sobre todo en el universitario, con el fin de intentar desafiarla, aunque no prohibiendo su utilización, sino más bien que los profesores junto con los directivos de las escuelas y universidades establezcan reglas de control para el buen uso del celular y diversos dispositivos electrónicos en clase, de tal manera que se colabore con el bienestar psicológico de los estudiantes universitarios, pues como ya se ha señalado, la solución no es prohibir su uso, sino más bien que se utilice de manera responsable.

\section{REFERENCIAS}

Barrios, D., Bejar, V., \& Cauchos, V. (2017). Uso excesivo de smartphones/ teléfonos celulares: Phubbing y Nomofobia. Revista Chilena de Neuropsiquiatría, 55(3), 205-206. Recuperado de: https://scielo.conicyt. cl/pdf/rchnp/v55n3/0034-7388-rchnp-55-03-0205.pdf

Capilla, E., \& Cubo, S. (2017). Phubbing. Conectados a la red y desconectados de la realidad. Un análisis en relación con el bienestar psicológico. Revista de Medios y Educación, 1(50), 173-185. Recuperado de: http://www.reda lyc.org/pdf/368/36849882012.pdf

Cisneros, L. \& Robles, S. (2017). ¿Para qué utilizan el celular en el aula, los estudiantes universitarios? XIV Congreso Nacional de Investigación Educativa (COMIE). San Luís Potosí, México.

Delena, M. (2014). Adicción tecnológica. Buenos Aires, Argentina: Universidad de Palermo. Recuperado de: http://www.adinarosario.com/fotos/bibliot eca/adicc10g.pdf

Esparza, D. (2016). ¿Comunicados o conectados? Recuperado de: ttps:// es.aleteia.org/2016/09/26/comunicados-o-conectados/

Felix, V., Mena, L., Ostos, R., \& Acosta, M. (2017). Nomofobia como factor nocivo para los hábitos de estudio. Revista de Filosofía y Cotidianidad, 3(6), 23-29. Recuperado de: https://www.researchgate.net/ publication/323933302_Nomofobia_como_factor_nocivo_para_los_habitos_de_estu

Gómez, J. (2013). El "phubbing", otra amenaza contra las relaciones interpersonales $y$ laborales. Recuperado de: https://www.merca20.com/el-Phubbing-otra-amenaza-contra-lasrelaciones-nterpersonales-y-laborales/

Guijosa, C. (2018). Los smartphones son una distracción en el salón de clase, según encuesta. Recuperado de: https://observatorio.tec.mx/edu-news/los-smar tphones-son-una-distraccion-en-el-salon-de-clase

González, J., Hernández, I., Martínez, R., Matos, J., Galindo, M., \& García, S. (2017). Interferencia de la tecnología en las relaciones de pareja y su impacto en la salud mental de la mujer puertorriqueña. Revista puertorriqueña de Psicología, 29(1), 56-71. Recuperado de: https://dialne t.unirioja.es/descarga/articulo/6496521.pdf 
Hertlein, K., \& Blumer, M. (2014). The Couple and Family Technology Framework: Intimate Relationships in a Digital Age. Recuperado de:

Shawab, K. (2016). The Fourth Industrial Revolution. Kindle Edition. Recuperado de: http://www3.weforum.org/docs/Media/KSC_4IR.pdf

Vargas, I., Fernández, M., \& Bauza, B. (2016). La comunicación pedagógica y su influencia en el proceso de enseñanza aprendizaje de la lengua española: principales problemas en los estudiantes de la carrera de educación primaria en Las Tunas (Cuba). Revista GeoGraphos, 7(86), 132-146. Recuperado de:

Villafuerte, C., \& Vera, M. (2019). Phubbing y género en un sector académico en Quito: Uso, abuso e interferencia de la tecnología 